\title{
Autonomous Identification and Planning System for Quadrotor UAV Based on Vision
}

\author{
Xuan DU, Kang ZHU, Bo QIN
}

College of Mechanical \& Power Engineering, China Three Gorges Univ., Yichang 443002, China

\begin{abstract}
Keywords: Quadrotor UAV, Monocular vision, Route planning, Embedded control.
\end{abstract}
\begin{abstract}
An autonomous identification and planning system is proposed and a flight platform based on Linux embedded control system is developed for a quadrotor unmanned aerial vehicle (UAV) based on vision. In the process of control, through the Zenmuse X3 camera and the Open CV library for real-time image processing, to achieve the UAV target identification and localization. By improved genetic algorithm to plan a reasonable path for the UAV, make its complete the mission from the starting point to the end point. The ultrasonic data from the Guidance is acquired, and the UAV accurate position can be realized by the acquired near-ground height information. The proposed approach is verified by experiments.
\end{abstract}

\section{Introduction}

At present, intelligent products are gradually changing people's production and lifestyle. Quadrotor unmanned aerial vehicle with vertical take-off and landing and other characteristics. Its application areas become more and more widely [1]. Typical products are DJI, XIRO, 3D Robotics in civil field.

One of the necessary conditions for UAV to stabilize flight control is to obtain the real-time motion state of the aircraft. Because the aircraft has the characteristics of flying in the air, limited load and high energy consumption, many methods of obtaining the state information of the traditional robot are not effective in some extent. In the most of works, laser range finder as an instrument for measuring the distance, it can detect the distance between the UAV and obstacles. It is more used in the UAV. But because of its limited accuracy, it is used in UAV indoor positioning and navigation [2]. In contrast, the machine vision is a more appropriate choice for the aircraft outdoor positioning flight. On the other hand, the camera is lighter than LIDAR and meets the load design requirements [3]. Xu-Chao CHEN uses the method of visual servo to study the hovering control of the UAV. Combining with the data of the inertial measurement unit, the aircraft dynamics model adopts the back-stepping method to design the controller to realize the hovering control of the aircraft [4]. Aiming at the autonomous flight control problem of unmanned aerial vehicles in the absence of GPS signal, Mei-Hui CAO designed a vision-based autonomous positioning and control method using improved SLAM algorithm, PID control and nonlinear controller [5]. Qiang LIU studied the problem of ground line structure in the indoor environment. The path recognition is accomplished by using the region of interest (ROI) of image, HSV color space threshold segmentation, Gaussian filtering and edge detection [6]. Bin DI considers the problem of allocating heterogeneous unmanned aerial vehicles to varied types of targets performing detection, attack and verification tasks. The model of the distributed task allocation for heterogeneous UAV is established. And the distributed auction algorithm using limited communications is developed to solve the task allocation problem optimally [7].

There are many researches on machine vision, path planning and PID control of aircraft. However, in the practical application of search, rescue, detection and transportation of UAV, image recognition, path planning and flight control are used integratedly to meet the functional requirements. In this paper, based on the Linux embedded control system architecture, image processing, object recognition and path planning are integrated on the Manifold computer, and the results are transferred to the UAV flight control system. This design avoids the dependence on the wireless data transmission link and eliminates the problems of system delay caused by wireless transmission. This paper designs an autonomous identification and planning system for quadrotor 
UAV based on vision, and builds the experimental platform of the aircraft. Combined with X3 camera and OpenCV library to complete the target object recognition and positioning. An improved genetic algorithm is used to efficiently complete the path planning problem of unmanned aerial vehicles. The precise flight control of the UAV from the starting point to the end point is realized by flight example.

\section{Target Identification and Location Technology Based on Vision}

Through the image processing operation can be completed in the field of view of the target object recognition, but the monocular camera model can't get the actual distance of the target object from the UAV. That is unable to complete the positioning of the target object. Therefore, in this paper, the UAV and ground height information acquired by Guidance vision navigation system and camera calibration are used to complete the target object location. Figure 1 shows the target identification, positioning process.

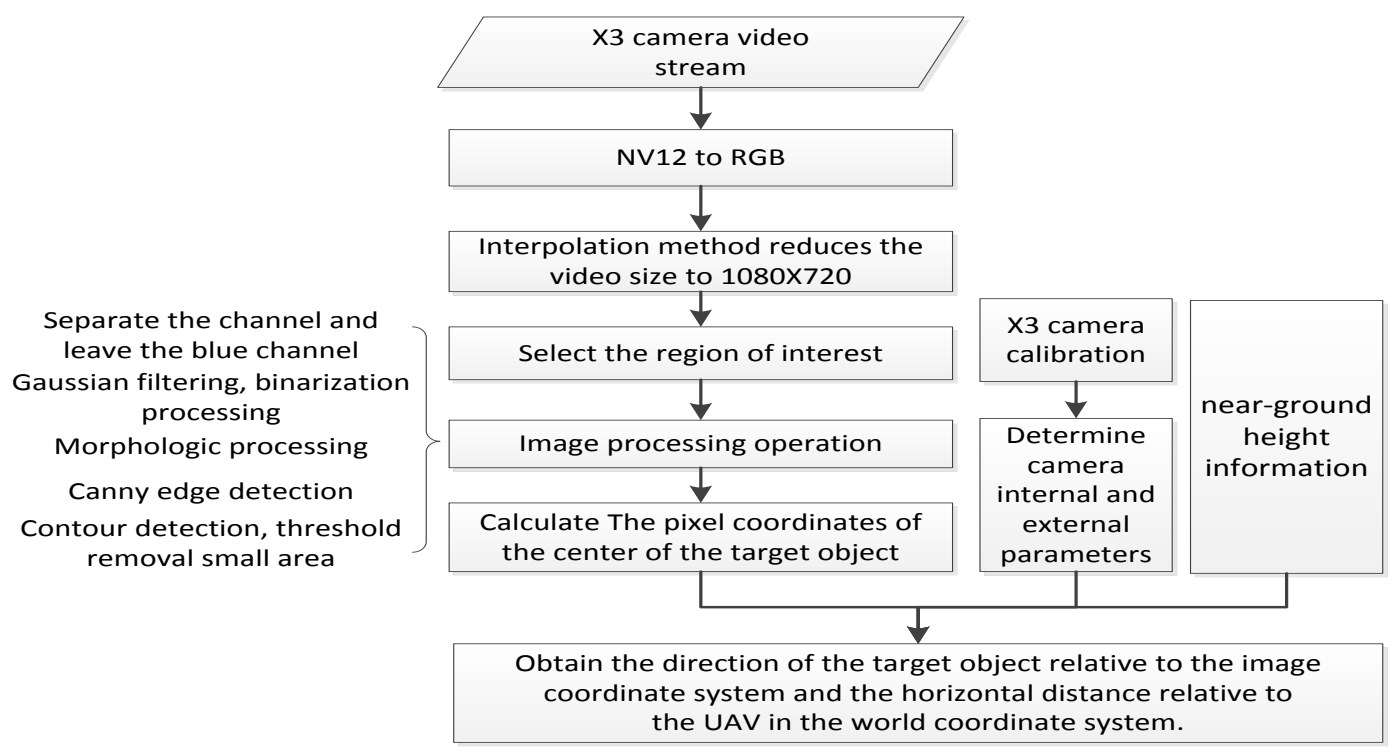

Figure 1. Target recognition and location

\section{Image Processing}

Through the X3 camera, Manifold computer to get the image size is $1280 \times 720$, encoding format is NV12. And the images needed for image processing should be color images, so need to adjust the image size and encoding format conversion, and then the target recognition process. In this paper, a specific visual algorithm is designed for specific experimental objects.

\section{NV12 Encoding to RGB Encoding}

Camera initialization, $\mathrm{X} 3$ camera video stream is stored in the form of one-dimensional string of NV12 encoding format. The Y, U, V components of the video are extracted from the string as follows:

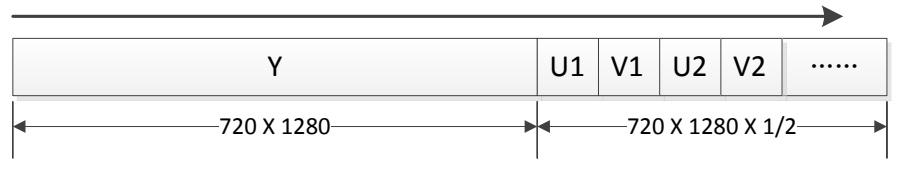

Figure 2. NV12 encoding format

The equation (1) gives the conversion of YUV to RGB: 


$$
\begin{aligned}
& R=Y+1.4075 *(V-128) \\
& G=Y-0.3455 *(U-128)-0.7169 *(V-128) \\
& B=Y+1.7790 *(U-128)
\end{aligned}
$$

Where, R, G, and B represent three channels of a color image. X3 camera color image information can be obtained through the channel fusion.

\section{Visual Algorithm Design}

Image processing is to get a clear outline of object in the image, easy to get the pixel center coordinates. In this paper, using image gray level, Gaussian filtering, gray image equalization as image preprocessing. Image threshold segmentation, morphological processing, canny edge detection and other methods to detect the target object.

The identification of campus road is taken as an example to show how the algorithm works. As shown in Figure 3.

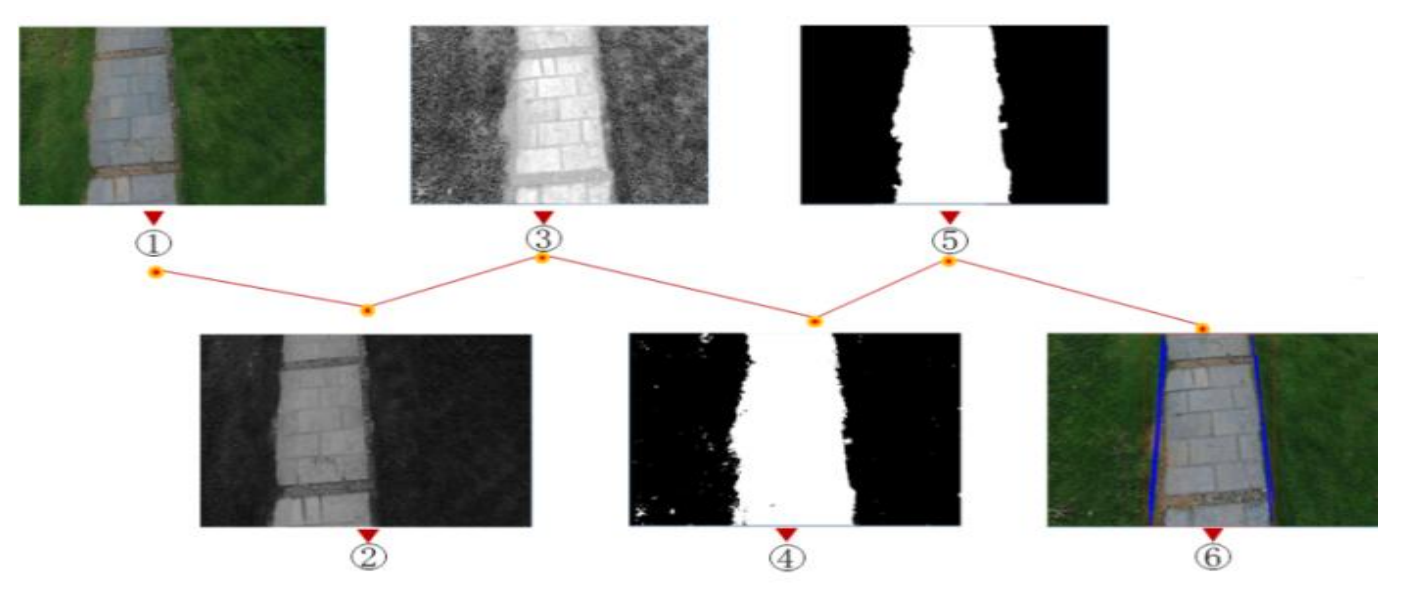

Figure 3. Image processing examples

In Figure 3, several important processes in image processing are represented in the form of a label. The label (1) indicates the restored color image, the label (2) indicates the gray image calculated by the weighted average method; the label (3) indicates the state after the image gray equalization; the label (4) is the state after the image threshold segmentation; he label (5) is the state after Canny edge detection; label (6) for the use of Hough linear transformation, this is the final test results.

\section{Target Location based on Sensor Data Fusion}

Through the above calculation can detect the target object, but cannot get the actual distance between the target and the aircraft. General binocular vision algorithm can get the three-dimensional coordinates of objects in space. Because Quadrotor UAV has the characteristics of nonlinear, strong coupling and static instability, so it has higher demand on real-time performance. While binocular vision algorithm is more computationally intensive and has a long processing time and is limited by computer hardware. Therefore, in this paper, the ultrasonic sensor in Guidance vision navigation module is used as the auxiliary information of position information, and the target location is achieved by monocular vision algorithm.

Camera calibration is the first step to monocular vision positioning, to provide the necessary camera parameters. In this paper, Zhang's calibration method based on planar chessboard is adopted. According to the camera imaging model can be obtained as follows equation [8].

$$
\mathrm{s} \tilde{m}=\mathrm{A}[\mathrm{R} \mathrm{t}] \tilde{M}
$$

Where the homogeneous coordinates of $m$ represent the pixel coordinates $(\mathrm{u}, \mathrm{v}, 1)$ of the image plane, the homogeneous coordinates of $\mathrm{M}$ represent world coordinate points $(\mathrm{X}, \mathrm{Y}, \mathrm{Z}, 1), \mathrm{R}$ is the rotation matrix, $\mathrm{t}$ is the translation matrix, $\mathrm{S}$ is the size factor. $\mathrm{A}$ is an internal reference of the 
camera, and is specifically shown as follows.

$$
A=\left[\begin{array}{ccc}
f / d_{x} & \gamma & u_{0} \\
0 & f / d_{y} & v_{0} \\
0 & 0 & 1
\end{array}\right]
$$

Where, $\alpha=\mathrm{f} / \mathrm{d}_{\mathrm{x}}$ (f is the camera focal length), $\beta=\mathrm{f} / \mathrm{d}_{\mathrm{y}}, \gamma$ represents the point's size of the deviation in the $x, y$ direction, the general value of 0 .

The camera calibration model is solved by computing the PnP (Perspective Points) problem [9]. X3 camera internal and external parameters can be calculated. Because this article is to obtain the target object relative to the aircraft's position and distance, the camera parameter $\mathrm{f}$ is mainly used. When the aircraft detects the target object, by the recognition algorithm can be obtained the target pixel coordinates $\left(\mathrm{u}_{1}, \mathrm{v}_{1}\right)$, according to the triangle rule.

$$
\left\{\begin{array}{l}
d_{x}=h * \frac{u_{1}}{f} \\
d_{y}=h * \frac{v_{1}}{f}
\end{array}\right.
$$

Where, $h$ is the near-ground height of the UAV, $f$ is the focal length of the camera. $\mathrm{d}_{\mathrm{x}}, \mathrm{d}_{\mathrm{y}}$ represent the horizontal distance and vertical distance of the target point from the center of the UAV platform.

\section{UAV Path Planning Technology}

\section{Path Planning Objective and Optimization Model}

UAV application scope is more and more widely, but these UAVs generally have an obvious problem - endurance is limited. Such as DJI M100 UAV in the case of Zero-load, use of TB47D battery, can only fly about 25 minutes. Therefore, planning a reasonable UAV route is extremely important. In this paper, based on the identification of all target points, established a path optimization model, and using an improved genetic algorithm to plan the UAV route, so as to improve the flight efficiency.

Suppose the UAV has $\mathrm{n}$ target points, the distance between the point $i$ and the point $j$ is expressed as $d_{i j}$. When the aircraft reaches the target point, it does not immediately stop, but slip forward for some distance due to inertia. In this paper, the adjustment distance brought by the characteristics of the aircraft is defined as $s_{i j}$. The size of $s_{i j}$ will be determined by $d_{i j}$. The multiple flight experiment results show that when the value of $d_{i j}$ is less than 3 meters, $s_{i j}$ is 0.5 ; otherwise, $s_{i j}$ is 1. The mathematical model of the aircraft path planning problem can be described as follows.

$$
\begin{aligned}
& \min \mathrm{f}=\sum_{\mathrm{i}=1}^{\mathrm{n}-1} \mathrm{~d}_{\mathrm{ij}}+\mathrm{s}_{\mathrm{ij}}+\mathrm{d}_{\mathrm{n} 1} \\
& \text { s.t. }\left\{\begin{array}{l}
d_{i j}>0 \\
s_{i j}>0 \\
j=i+1
\end{array}\right.
\end{aligned}
$$

Where $i$ is the number of the target point, $i=1,2,3, \ldots, n ; \mathrm{d}_{\mathrm{ij}}$ is the distance between two target points, and $d_{n 1}$ is the distance from the end point to the starting point.

\section{Improved Genetic Algorithm}

\section{Chromosome Coding}

The choice of coding mode is the key to solving the problem by genetic algorithm. The coding method adopted in this paper is path representation. This representation is the most direct and natural representation of the TSP problem, in which the target points are arranged in order of access. The code string $\left(\mathrm{x}_{1}, \mathrm{x}_{2}, \ldots \mathrm{x}_{\mathrm{n}}\right)$ represents the start from the target point $\mathrm{x}_{1}$, passing through the points $x_{2}, \ldots x_{n}$, and finally to the starting point $x_{1}$. This representation not only satisfies the 
constraints of the TSP problem, that is, it guarantees that each target point passes once and only once, and ensures that any subset does not form a loop.

\section{Initialization and Fitness Function}

The purpose of population initialization is to generate the first generation population and to guarantee the quality and diversity of the population. The initialization method adopted in this paper is the nearest neighbor ( $\mathrm{k}$-th nearest neighbor) method. Initialization is as follows: Specify the starting point of the flight, and according to the nearest neighbor method to find the next point. And then the current point as a starting point, again using the nearest neighbor method to find the next point, until all the target points are accessed.

The fitness function is used to evaluate the advantages and disadvantages of genetic individuals. The shorter the selected path, the greater the fitness function value.

\section{Crossover and Mutation}

The key of using genetic algorithm to solve the path planning problem is to ensure the efficiency of the newly generated individuals in the search process by using crossover and mutation.

In this paper, the crossover method is as follows: a binary chain is generated at random and its length is the same as the initial population length. The gene with $l$ in the parent $\mathrm{P}_{\mathrm{i}}$ sequence is copied to the corresponding position of the children $S_{i}$. The gene with $O$ in the $P_{i}$ sequence is copied to the corresponding position in the children $S_{j}$. The genes with $O$ in the parent $P_{i}$ sequence are added to the corresponding vacancies in the progeny $S_{i}$ as the order in which they appear in the parent $P_{j}$; the gene with $l$ in the parent $P_{j}$ sequence are added to the corresponding vacancies in the children $S_{j}$ as the order in which they appear in the parent $P_{i}$.

In this paper, multi-point mutation operator is used. The basic idea of multi-point mutation is to randomly generate $n$ points in the parent. The $n$ points are rearranged to form $n !-1$ new individuals.

\section{Algorithm Implementation Steps}

1) Initialization, set the population size $N$, genetic algebra $T$, crossover probability $\mathrm{P}_{\mathrm{c}}$, mutation probability $\mathrm{P}_{\mathrm{m}}$.

2) Determine whether to meet the termination conditions, meet the direct end, or go to the next step.

3) Calculating the fitness function value of individual in the population.

4) Generating new individuals by genetic manipulation such as selection, crossover and mutation.

5) Calculate the fitness function values of the parent population and all offspring individuals. According to the value of fitness function, select a part as the next generation parent $\mathrm{P}(\mathrm{t})$.

6) The genetic algebra $T$ is increased by 1 . Determine whether to meet the termination conditions, meet to stop the iteration, or continue to calculate the individual fitness value.

7) Repeat steps 4) 6) until the hereditary algebra reaches $T$, get the optimal solution and output the result.

\section{Flight Experiment and Result Analysis}

\section{Build Flight Platform}

Based on the above theoretical analysis, this paper builds an experimental platform based on Linux embedded control framework. Manifold computer equipped with NVIDIA's low-power high-performance embedded chip Tegra K1 as the core processor, with up to 326GFLOPS computing power. The platform can perform the relevant visual algorithm well, and can realize the autonomous flight control of the aircraft without ground station.

Experimental flight platform equipped with the main structure, including remote control, embedded computers, external cameras, enhanced wireless network card. The flight platform is shown in Figure 4.

1) DJI launched the developer kit M100, the N1 flight control board integrates a barometer, 
inertial navigation unit.

2) Manifold Embedded Computing with low power NVIDIA Kepler ${ }^{\mathrm{TM}}$ Geforce ${ }^{\circledR}$ image processor.

3) DJI Zenmuse X3 camera, the maximum resolution of $4096 \times 2160$, support multi-frame image noise reduction.

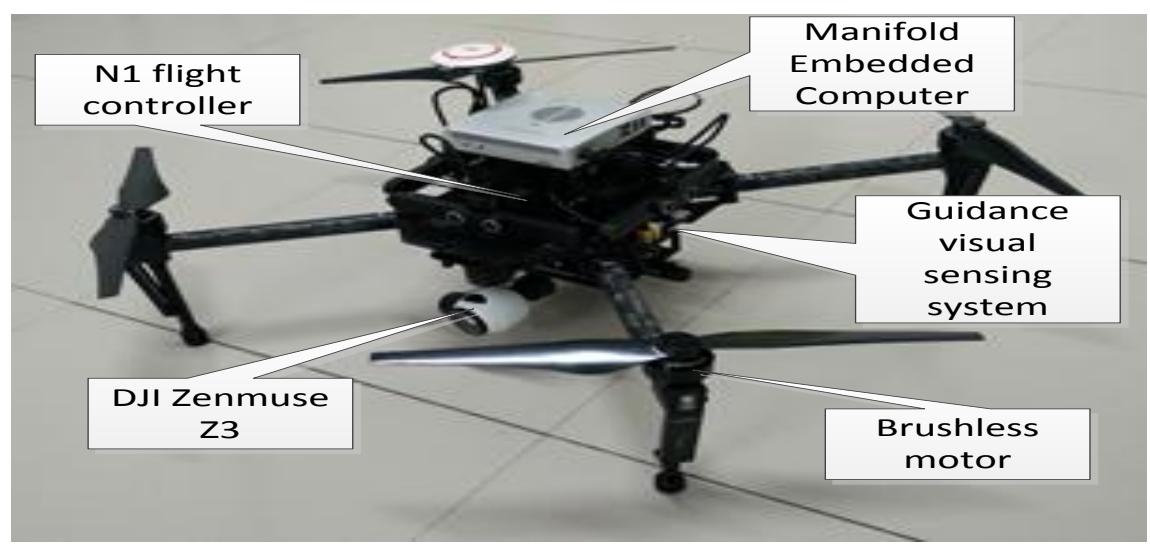

Figure 4. Quadrotor UAV experimental platform

UAV intelligent target recognition and path planning system is shown in Figure 5. When the UAV is started by the remote control, the information collected by the camera and the ultrasonic sensor is processed by the corresponding visual processing module and Guidance vision sensing navigation system respectively. The data is returned to the onboard manifold computer and the target identification and path planning related operations are completed. Through the UART2 serial port, the data transmitted to the flight controller N1. N1 control electronic governor to adjust the speed of four brushless motor, and ultimately control the UAV to complete their own missions.

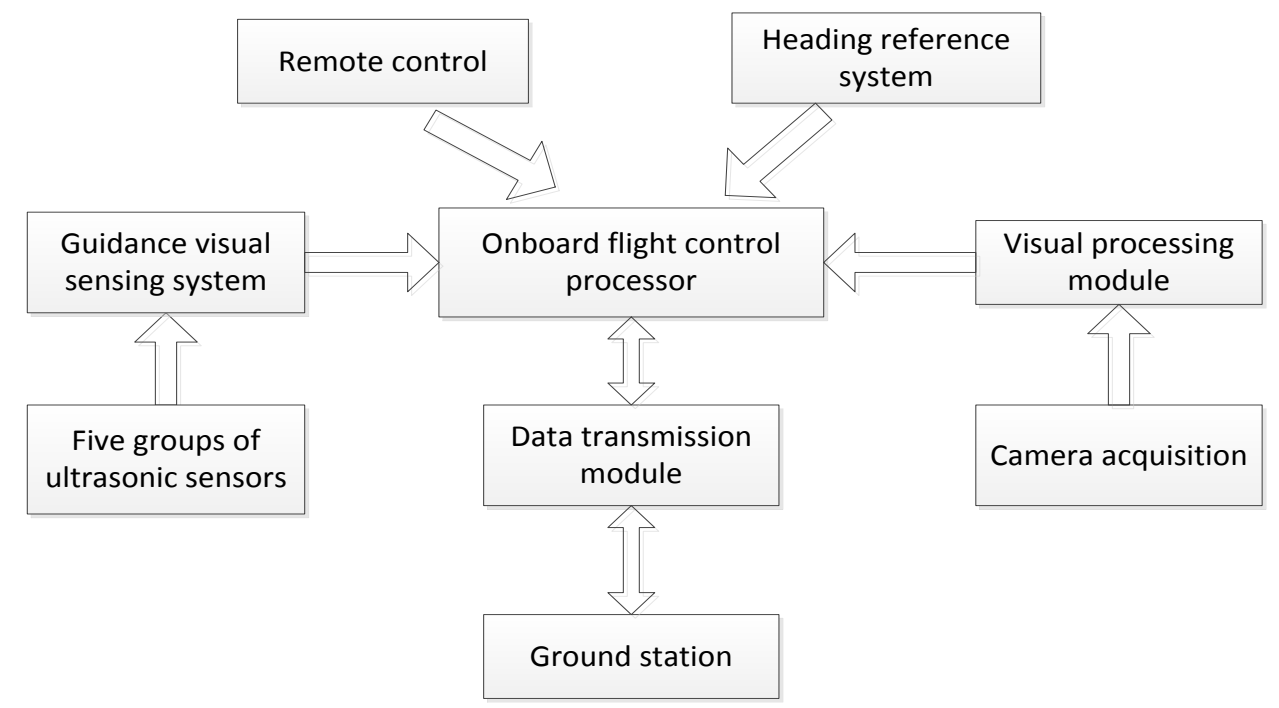

Figure 5. Integrated Design of Embedded System

\section{Flight Experiment}

The flight experiment is shown in figure 6. The basic process of flight experiment: the aircraft placed in front of the starting point, and adjust the X3 camera lens down. Switch to the automatic flight mode when the UAV goes up to see all target points through the X3 camera. In this case, the target recognition, positioning, path planning and flight control tasks designed in this paper are completed automatically by the aircraft. 


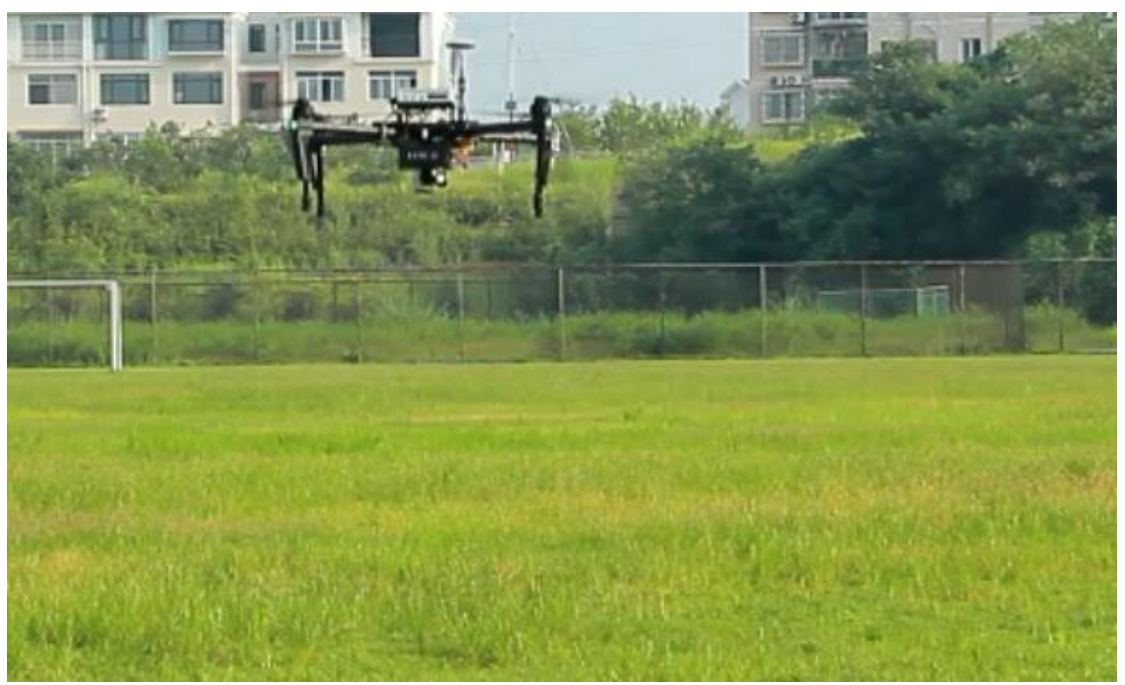

Figure 6. Outdoor flight experiment of Quadrotor UAV

Figure 7 shows: The visual processing flow in UAV flight.

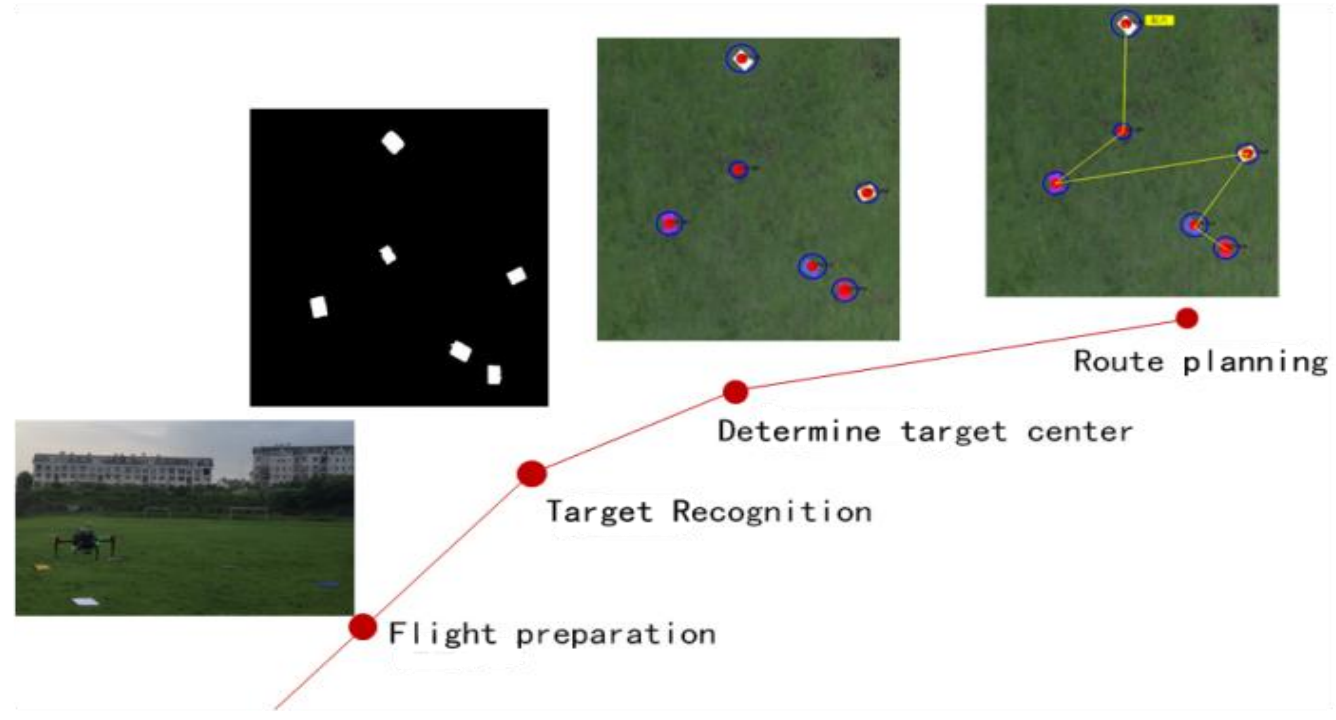

Figure7. Flight experiments

\section{Conclusions}

The main purpose of this paper is the intelligent flight of Quadrotor UAV. The aircraft vision recognition and positioning algorithm, the aircraft path planning method, the aircraft near - ground height control scheme are designed and the experiment platform of the aircraft is successfully established.

(1) Completing the conversion of the video stream format, design the image processing technology under the platform of the aircraft, and realize the recognition and localization of the target in the visible area.

(2) Through the improved genetic algorithm, the crossover and mutation operator are designed. Solving the optimal path of UAV flight to ensure that the task of the UAV from the starting point to finish and through all the intermediate points.

(3) According to the parameters of camera calibration and Guidance visual sensing navigation system, the relative distance of the target object from the UAV center is calculated. Thus ensure the accuracy of UAV altitude information.

(4) Linux-based embedded control system, with Tegra K1 chip to improve the real-time. So that UAV has a fully autonomous and complete a certain task. 


\section{References}

1. Gupte S. Mohandas P I T, Conrad J M. A survey of quad-rotor unmanned aerial vehicles[C] // Proc. of Proceedings of IEEE Southeast Con. Orlando: IEEE, 2012: 1-6

2. SHEN S, MICHAEL N, KUMAR V. Autonomous multi-floor indoor navigation with a computationally constrained MAV[C]// proceedings of the 2011 IEEE International Conference on Robotics and Automation, Shanghai, China, 2011: 20-25

3. Bin XIAN, Yang LIU, Xu ZHANG. Autonomous control of a micro Quadrotor unmanned aerial vehicle using optical flow [J]. Journal of mechanical engineering, 2015, 51(9): 58-59

4. Xu-Chao CHEN, Zhi-Qiang Cao, Ying-Ying YU. Hovering control for quadrotor helicopter based on visual servo [J]. J.Hua Zhong Univ. of Sci. \& Tech. (Natural Science Edition), 2015, 43: 6-7

5. Me-Hui CAO, Bin XIAN, Xu ZHANG. An autonomous vision-based localization and control system for quadrotor UAV [J]. Information and Control, 2015, 44(2): 190-191

6. Qiang LIU, Pei-Pei NI, Feng LIU. Path-Following of Quadrotor Aircraft Based on Vision [J]. Journal of Sichuan Ordnance, 2014,35(11): 106-107

7. Bin DI, Rui ZHOU, Quan-Xin DING. Distributed coordinated heterogeneous task allocation for unmanned aerial vehicles [J]. Control and decision, 2013, 28(2): 274-276

8. Zheng-You ZHANG. Flexible camera calibration by viewing a plane from unknown orientations. //The Proceedings of the Seventh IEEE International Conference on Computer Vision. 1999

9. Lepetit V, Moreno-Noguer F, FuaP. EPnP: An accurate $O$ (n) solution to the PnP problem [J]. International Journal of Computer Vision. 2009,81(2):155-166 